\title{
Pembrolizumab in the Treatment of Patients with Microsatellite Unstable Colorectal Cancer
}

\author{
Diana Maria Leite da Cunha Russo* and Francisco Miguel Costa Silva Mendes \\ Faculty of Medicine, University of Porto, Portugal
}

*Corresponding author: Diana Maria Leite da Cunha Russo, Rua Nova dos Fraiões, Faculty of Medicine of University of Porto, Portugal.

To Cite This Article: Diana Maria Leite da Cunha Russo, Francisco Miguel Costa Silva Mendes, Pembrolizumab in the Treatment of Patients with Microsatellite Unstable Colorectal Cancer. Am J Biomed Sci \& Res. 2021 - 11(6). AJBSR.MS.ID.001692. DOI: 10.34297/AJBSR.2021.11.001692.

Received: 䟧 January 06, 2020; Published: 阱 February 09, 2021

\begin{abstract}
Context
Colorectal cancer is one of the most common tumours in the world, with microsatellite instability having an important role in the development of these tumours. Microsatellite instability is responsible for the worse response of tumours to chemotherapy, while also having a role in the better prognosis associated with these tumours. This relates to the higher immune infiltration that results from the high neoantigen load of microsatellite unstable tumours. This highly responsive immune microenvironment seen in microsatellite unstable colorectal cancer makes these tumours great candidates for therapies targeting the immune system, such as immune checkpoint inhibitors. The PD-1/PD-L1 axis is upregulated in colorectal cancer with microsatellite instability, making the blockade of this axis a good therapy alternative for these patients.
\end{abstract}

Pembrolizumab has shown to be an effective PD-1 blocker, with trials showing good responses to this therapy, as seen in the most recent KEYNOTE-177 trial.

Conclusion: Colorectal cancer with microsatellite instability is a subtype of tumour with characteristics making it a great candidate for the treatment with immunotherapy, namely with the PD-1 blocker pembrolizumab, enhancing the importance of including this treatment in the options available for these patients.

Keywords: Pembrolizumab; Colorectal Cancer; Microsatellite Instability; Immunotherapy; Treatment

Abbreviations: CRC: Colorectal Cancer; ICI: Immune Checkpoint Inhibitor; DMMR: Deficient Mismatch Repair; MSI: Microsatellite Instability; ORR: Overall Response Rate; OS: Overall Survival; PFS: Progression Free Survival; 5-FU: 5-fluorouracil

\section{Introduction}

Colorectal cancer is one of the most common tumours in the world in both men and women, while chemotherapy and surgery remain the standard of care for these patients [1] Microsatellite instability is responsible for about $15 \%$ of these tumours, with $5 \%$ of all CRC being associated with hereditary MSI [2]. While MSI is typically associated with a better prognosis and longer OS, it is also known to be a predictor of poorer response to chemotherapy with 5-FU derivates [3], fluoropyrimidines, platinum compounds and methylating agents [4] MSI is responsible for a high lymphocyte infiltration in the tumour microenvironment, which makes these tumours good candidates for immune based therapy [5], such as the PD-1 blocker pembrolizumab.

\section{Colorectal cancer with microsatellite instability}

Microsatellite instability is responsible for a great variability in microsatellites' size in the patients' genome, associated with DNA mutations and high neoantigen production, facilitating the recognition of the tumour by the host immune system [6]. MSI CRC is usually associated with younger age of onset, proximal located tumours and a high lymphocytic reaction, as well as a lower risk of nodal and distant metastases [7]. These tumours have a highly active immune microenvironment, with high CD8+ T-cells activation and an upregulation of immune checkpoints such as PD-1/PD-L1, favouring this checkpoint as a potential therapeutic target [1]. 
The PD-1/PD-L1 signalling axis is upregulated in microsatellite unstable CRC, impairing the antitumour immune response [8] MSI is associated with a high number of tumour infiltrating lymphocytes that are PD-L1 positive, which in turn is associated with a good prognosis [9]. Furthermore, the number of DNA replication errors present in MSI tumours are recognized by the host immune cells as foreign, resulting in a high immune infiltration (mainly CD8+ T-cells), favouring the response of these tumours to ICI.8, [10]. Several studies have been conducted in order to assess the response of MSI CRC to immunotherapy, such as PD-1 and PD-L1 blockers (such as pembrolizumab and nivolumab, both PD-1 blockers), showing good responses to these therapies [11].

\section{Pembrolizumab in the treatment of CRC with MSI}

Pembrolizumab is a humanized monoclonal IgG4 antibody which binds to PD-1, blocking the interactions of the PD-1/PDL1 axis, enhancing the immune recognition of the tumour and facilitating the subsequent response [11]. Clinical trials such as KEYNOTE-016, KEYNOTE-164 and KEYNOTE-028 have shown favourable outcomes of patients with MSI tumours regarding the OS, ORR and PFS in the patient groups treated with pembrolizumab [12-14]. In the most recent KEYNOTE-177 trial, patients with MSI or dMMR with previously untreated CRC were randomly assigned to receive pembrolizumab or chemotherapy (crossover from the chemotherapy group to the pembrolizumab group was allowed if disease progression was observed). The primary endpoints were PFS and OS with a median follow-up of 32.4 months [15].

Pembrolizumab was proven superior to chemotherapy mainly regarding PFS (16.5 months vs. 8.2 with chemotherapy, $p=0.0002$ ). Besides that, after 24 months of follow-up, the mean survival was 13.7 months in the pembrolizumab treated group, against 10.8 months in the chemotherapy group [15]. Furthermore, there was a higher overall response in the pembrolizumab group (43.8\%) than in the chemotherapy group (33.1\%). At 24 months there were $83 \%$ of patients with ongoing response in the pembrolizumab group, with only $35 \%$ of patients in the chemotherapy group [15]. Regarding adverse effects, the chemotherapy group has also shown to have greater rates of side effects (66\% vs. $22 \%$ ) than the group treated with pembrolizumab [15].

\section{Conclusion}

CRC is one of the most common tumours in the world, with MSI having an important role in both the development of these tumours and their response to the available therapies, making the response to chemotherapy worse than in other CRC subtypes. MSI is also responsible for a characteristic tumour microenvironment which favours these tumours' response to immunotherapy, mainly through the blockade of the PD-1/PD-L1 axis, such as the PD-1 blocker pembrolizumab. On that note, clinical trials show the good response rates and improvement in OS of patients with MSI CRC treated with pembrolizumab, making this the rationale for the implementation of this antibody in the treatment of CRC with MSI.

\section{Acknowledgement}

None.

\section{Conflict of Interest}

There are no conflicts of interest to declare.

\section{References}

1. Oliveira AF, Bretes L, Furtado I (2019) Review of PD-1/PD-L1 Inhibitors in Metastatic dMMR/MSI-H Colorectal Cancer. Front Oncol 9: 396.

2. Colle R, Cohen R, Cochereau D, Duval A, Lascols O, et al. (2017) Immunotherapy and patients treated for cancer with microsatellite instability. Bull Cancer 104(1): 42-51.

3. Gupta R, Sinha S, Paul RN (2018) The impact of microsatellite stability status in colorectal cancer. Curr Probl Cancer 42(6): 548-559.

4. Viale G, Trapani D, Curigliano G (2017) Mismatch Repair Deficiency as a Predictive Biomarker for Immunotherapy Efficacy. Biomed Res Int 2017: 4719194.

5. Gelsomino F, Barbolini M, Spallanzani A, Pugliese G, Cascinu S (2016) The evolving role of microsatellite instability in colorectal cancer: A review. Cancer Treat Rev 51: 19-26.

6. Zhao P, Li L, Jiang X, Li Q (2019) Mismatch repair deficiency/microsatellite instability-high as a predictor for anti-PD-1/PD-L1 immunotherapy efficacy. J Hematol Oncol 12(1): 54.

7. Chang L, Chang M, Chang HM, Chang F (2017) Expending Role of Microsatellite Instability in Diagnosis and Treatment of Colorectal Cancers. J Gastrointest Cancer 48(4): 305-313.

8. Kim JH, Park HE, Cho NY, Lee HS, Kang GH (2016) Characterisation of PD-L1-positive subsets of microsatellite-unstable colorectal cancers. Br J Cancer 115(4): 490-496.

9. Ho HL, Chou TY, Yang SH, Jiang JK, Chen WS, et al. (2019) PD-L1 is a double-edged sword in colorectal cancer: the prognostic value of PDL1 depends on the cell type expressing PD-L1. J Cancer Res Clin Oncol 145(7): 1785-1794.

10. Franke AJ, Skelton WP, Starr JS, Parekh H, Lee JJ, et al. (2019) Immunotherapy for Colorectal Cancer: A Review of Current and Novel Therapeutic Approaches. J Natl Cancer Inst 111(11): 1131-1141.

11. Arora SP, Mahalingam D (2018) Immunotherapy in colorectal cancer: for the select few or all? J Gastrointest Oncol 9(1): 170-179.

12. Ciardiello D, Vitiello PP, Cardone C, Martini G, Troiani T, et al. (2019) Immunotherapy of colorectal cancer: Challenges for therapeutic efficacy. Cancer Treat Rev 76: 22-32.

13. Morse MA, Hochster H, Benson A (2020) Perspectives on Treatment of Metastatic Colorectal Cancer with Immune Checkpoint Inhibitor Therapy. Oncologist 25(1): 33-45.

14. O Neil BH, Wallmark JM, Lorente D, Elez E, Raimbourg J, et al. (2017) Safety and antitumor activity of the anti-PD-1 antibody pembrolizumab in patients with advanced colorectal carcinoma. PLoS One 12(12): e0189848.

15. Andre T, Shiu KK, Kim TW, Jensen BV, Jensen LH, et al. (2020) Pembrolizumab in Microsatellite-Instability-High Advanced Colorectal Cancer. N Engl J Med 383(23): 2207-2218. 